\title{
MEDO, PERCEPÇÃO DE RISCO E USO DE PRESERVATIVOS
}

\section{FEAR, RISK PERCEPTION AND CONDOM USE}

\author{
Ricardo Teixeira Veiga \\ Professor titular da Faculdade de Ciências Ecônomicas da Universidade Federal de Minas Gerais FACE/UFMG \\ Belo Horizonte, MG, Brasil \\ Email: ricardo.necc@gmail.com \\ Agnaldo Higuchi \\ Professor adjunto da Universidade Federal dos Vales do Jequitinhonha e Mucuri \\ Teófilo Otoni, MG, Brasil \\ Email: agnaldo.higuchi@gmail.com
}

Cátia Fabíola Parreira de Avelar

Doutoranda pelo Centro de Pós Graduação e Pesquisas em Administração da Universidade Federal de Minas Gerais - CEPEAD/UFMG Belo Horizonte, MG, Brasil

Email: catiaavelar@yahoo.com.br

\section{RESUMO}

$\mathrm{O}$ aumento do número de pessoas infectadas com o vírus HIV em muitas regiões brasileiras entre 2006 e 2016 mostra que houve uma redução nos comportamentos de prevenção da Aids. A fim de apoiar futuras campanhas de saúde, este trabalho teve como objetivo examinar a efetividade da manipulação do medo em uma propaganda e a importância do traço de personalidade Amabilidade na avaliação do risco de fazer sexo sem usar preservativo. Assim, realizamos um quaseexperimento com universitários de duas universidades brasileiras $(\mathrm{N}=92)$, aleatoriamente divididos em dois grupos, submetidos a duas versões diferentes de um anúncio impresso, composto por uma mensagem escrita informando a taxa de infecção por HIV / Aids em todo o mundo, seguida ou não por duas imagens de pessoas com Aids, uma delas mostrando um paciente terminal. $\mathrm{O}$ nível do medo evocado, o risco percebido e o traço de Amabilidade do sujeito experimental foram medidos. Uma análise ANCOVA mostrou que o anúncio mais emocional (aquele com imagens) provocou mais medo nos entrevistados. Além disso, foi detectado um efeito estatisticamente significativo do medo na percepção de risco. No entanto, não houve relação entre Amabilidade e risco percebido.

Palavras-chave: Resposta ao medo. Amabilidade. Aids. Uso de preservativo. Programas de Saúde Pública.

\section{ABSTRACT}

The increase AIDS infected people in many Brazilian regions between 2006 and 2015 shows that there has been a reduction in Aids prevention behaviors. In order to support future healthcare campaigns, this paper aimed to examine the effectiveness of the manipulation of fear in an advertisement, and the importance of the personality trait Agreeableness in assessing the risk of having sex without put on a condom. So, we carried out a quasi-experiment with undergraduates from two Brazilian universities $(N=92)$, randomly assigned to two groups, submitted to two different versions of a printed ad, composed of a written message informing the rate of HIVIAIDS infection worldwide, followed or not by two images of AIDS-infect people, one of them showing a terminal patient. The evoked fear level, the perceived risk, and the Agreeableness trait of the experimental subject were measured. An ANCOVA analysis showed that the most emotional ad (the one with images) elicited more fear in the respondents. Also, a statistically significant effect of fear on risk perception was detected. However, there was no relationship between Agreeableness and perceived risk.

Keywords: Fear response. Agreeableness. Aids. Condom use. Public health programmes. 


\section{INTRODUÇÃO}

O marketing social busca influenciar o comportamento voluntário de um público-alvo, de modo a substituir seus comportamentos de risco por outros mais saudáveis ou que levem a maior bem-estar individual ou coletivo (ANDREASEN, 1994). Vários estudos sugerem que o marketing social amadureceu como disciplina, destacando seu impacto positivo na sociedade (ROCHA et.al, 2017).

De fato, o marketing social é uma alternativa importante para tratar de questões relativas à saúde e bem-estar social, por exemplo, promover a adoção de dietas saudáveis, aumentar a atividade física regular e combater o consumo abusivo de tabaco, drogas ilícitas e álcool (WETTSTEIN; SUGGS; LELLIG, 2012; para uma revisão consultar GORDON et al., 2006), Além disso, o marketing social pode efetivamente diminuir o preconceito e a discriminação de pessoas HIV positivas (THAINIYOM; ELDER, 2017), estimular o anticonsumismo e promover o consumo sustentável (PEATTIE; PEATTIE, 2009), bem como apoiar a conservação de parques (LAl et al. 2009) etc.

Rocha et al. (2017) realizaram a revisão de estudos brasileiros sobre marketing social, encontrando 50 artigos, sendo 16 trabalhos sobre saúde pública (percepção de medicamentos genéricos, aleitamento materno, promoção do programa saúde da mulher, promoção da doação de sangue, medula óssea e órgãos, intenção de fazer dieta e exercícios físicos, uso de álcool e direção), 14 trabalhos explorando os conceitos de marketing social, 10 sobre responsabilidade social, 6 trabalhos abordando estratégia empresarial vinculada à utilização de marketing social e 3 trabalhos sobre o meio ambiente. Sagaz \& Lucietto (2016) verificaram que, no Brasil, o marketing social vem sendo aplicado na gestão de programas de saúde coletiva, melhoria de infraestrutura e qualidade de vida, em áreas como: habitação, educação, ecologia, gerenciamento de resíduos, poluição, violência, pobreza, trânsito, incentivo a boas práticas de higiene, controle da natalidade, diminuição da mortalidade infantil, uso de preservativos e doação de sangue, além do fomento de estilos de vida saudáveis, estimulando a adoção de padrões de alimentação saudáveis, o combate às drogas, a diminuição do consumo de tabaco e de bebidas alcoólicas.

Em marketing social, as propagandas são usadas não apenas para aumentar consciência sobre determinados riscos, mas também para mudar o comportamento e, para alcançar tal objetivo, a criação da mensagem é um fator crítico para persuadir e provocar mudança comportamental (D'SOUZA; TAY, 2016). Na saúde, campanhas de comportamento responsável ou seguro muitas vezes empregam comunicações com apelos emocionais, para evocar nas pessoas reações que as levem à mudança voluntária de hábitos ou adoção de condutas recomendadas (ANDREASEN, 2002).

No meio acadêmico, o poder persuasivo de campanhas e anúncios baseado no medo, seja para criar consciência ou para mudança de comportamentos, tem sido tópico de constante debate, já que nem todas as mensagens baseadas no medo afetam diferentes pessoas da mesma maneira (D'SOUZA; TAY, 2016). Por exemplo, a resposta ao estímulo do medo varia com o tipo de comunicação e com diferenças individuais no público (HASTINGS; SAREN, 2003), bem como com o grau de envolvimento e conhecimento do público-alvo sobre o tema (MOTA et al., 2013).

Para alguns autores, quanto maior o medo suscitado, maior o efeito persuasivo (HASTINGS; STEAD; WEBB, 2004). Por isso, é comum acreditar que comunicações ameaçadoras, com apelos emocionais mais fortes, causem mudanças de comportamento mais duradouras. Porém, pesquisa sobre o impacto do apelo 
ao medo, através da exposição de imagens sanitárias, para combater o tabagismo (Mota, et al., 2013), como as imagens usadas no país nas embalagens de maços de cigarro, desde 2001, mostrou que um apelo mais moderado pode ser mais eficaz, corroborando uma linha de estudos que sustenta que apelos muito fracos podem não surtir efeito, e excessivamente fortes podem ser contraproducentes, por levarem a um comportamento de fuga ou evitação.

Diferenças individuais, como traços de personalidade, também podem influir na resposta ao medo. $O$ traço Amabilidade, por exemplo, é um antecedente estatisticamente significativo da resposta ao medo em comunicações relacionadas aos riscos da direção desatenta (MOWEN; HARRIS; BONE, 2004). Por isso, para elaborar campanhas eficazes, que alertem sobre os riscos para saúde de condutas imprudentes, as autoridades públicas devem conhecer os fatores de persuasão, levando em conta as características de cada segmento-alvo (RUCKER; PETTY, 2006), e a eficácia do uso do apelo ao medo na comunicação.

Embora não haja uma análise clara sobre como as diferenças individuais influenciam as respostas ao medo, Mowen, Harris e Bone (2004) indicaram que traços individuais de personalidade podem influenciar significativamente as respostas do medo. Em função desse resultado e devido à controvérsia acerca da efetividade do uso do medo em campanhas que buscam a mudança de comportamento, o objetivo deste trabalho é examinar a efetividade do nível de medo evocado num anúncio impresso e do traço Amabilidade na avaliação de risco de não usar preservativo durante relações sexuais.

De forma preliminar e exploratória, procuramos contribuir teoricamente na elucidação se a percepção de risco da prática do sexo inseguro é influenciada por mensagens com apelos ao medo e por traços de personalidade. Especificamente na prática, compreender como o medo e a personalidade influenciam o uso de preservativos pode ajudar na criação de mensagens ou de elementos da mensagem que aumentem a percepção do risco de manter relações sexuais sem preservativos, incentivando seu uso, contribuindo para diminuir a propagação de doenças sexualmente transmissíveis (DST's), como a Aids.

\section{REFERENCIAL TEÓRICO}

\section{Marketing social}

A ciência de marketing teve seu escopo aumentado e evoluiu ao longo dos anos, mudando o seu entendimento e diversificando suas subáreas de conhecimento, estudando as relações de troca, levando em conta seu contexto (BASTOS; COSTA; VASCONCELOS, 2017). Dessa forma os estudos de marketing passaram de uma orientação tipicamente comercial para uma orientação social.

Segundo Schneider e Luce (2014) e Rocha et al. (2017), o marketing social originou-se de estudos iniciais da década de 1960 e em 1971 o termo "marketing social" foi estabelecido, partir do artigo de Kotler e Zaltman, "Social Marketing: An Approach to Planned Social Change". Kotler e Lee (2008, p. 207) definiram marketing social como "o uso dos princípios e técnicas de marketing para influenciar um público-alvo a voluntariamente aceitar, rejeitar, modificar ou abandonar um comportamento para o benefício de indivíduos, grupos ou da sociedade como um todo". Andreasen (1994, p.1 10) reposicionou o marketing social, conceituando-o como "a adaptação de tecnologias de marketing comercial a programas destinados a influenciar o comportamento voluntário dos públicos-alvo para melhorar o seu bem-estar pessoal e da 
sociedade da qual fazem parte". Tais mudanças de concepção são naturais. Schneider e Luce (2014) observam que o conhecimento é dinâmico e nem mesmo o conceito de marketing definido pela American Marketing Association é definitivo, passando por reavaliações periódicas com a finalidade de aproximar a teoria à prática profissional.

Buscam-se por meio de campanhas de marketing social, mudanças sociais que beneficiem a saúde pública, afetando os hábitos de consumo e o comportamento social, em prol do consumidor ou da sociedade em geral (BASTOS; COSTA; VASCONCELOS, 2017).

Em termos amplos, o objetivo do Marketing social é conscientizar ou sensibilizar as pessoas a respeito de um tema de natureza social e buscar uma mudança de comportamento social (MEIRA;SANTOS, 2012); sua missão é promover mudanças sociais desejáveis, de forma duradoura (DONOVAN; HENLEY, 2010). Entre as várias áreas de aplicação do marketing social estão a erradicação da miséria, promoção da saúde, melhoramento das condições sociais e reversão da situação de insustentabilidade (LEFEBVRE, 2013).

Para buscar promover mudanças sociais desejáveis e duradouras, é necessário o conhecimento do comportamento do público-alvo das campanhas, assim como dos meios mais efetivos para alcançar os objetivos propostos como, por exemplo, a redução do tabagismo (MOTA, et al. 2013); o aumento de doações de sangue (CUNHA; DIAS, 2008); redução do consumo de bebidas alcoólicas (BASTOS; COSTA; VASCONCELOS, 2017); a redução do preconceito e discriminação de pessoas HIV positivas (THAINIYOM; ELDER, 2017) e a promoção do uso de contraceptivos na adolescência (HILDEBRAND; VELOSO, 2012).

Obstáculos à implantação de programas de marketing social incluem a dificuldade de definir claramente qual é o problema gerencial, a multicausalidade e a interdependência com outros problemas, a incapacidade de antecipar completamente os desdobramentos das ações de um programa, a complexidade social e o fato de se exigir do público-alvo, em certos casos, ações consideradas desagradáveis e constrangedoras (LEFEBVRE, 2013).

Dados da Secretaria de Vigilância em Saúde sobre doenças sexualmente transmissíveis, Aids e hepatites virais mostram que, no período de 2007 até junho de 2017, o Sistema de Informação de Agravos de Notificação (SINAN), registrou 194.217casos de infecção pelo vírus HIV no Brasil, sendo 49,7\% \% (o maior percentual) deles na região Sudeste. A maior parte dos casos de infecção por HIV encontra-se nas faixas de 20 a 34 anos, com percentual de 52,5\% dos casos (MINISTÉRIO DA SAÚDE, 2017). Os dados indicam também um crescimento no número de casos no entre 2006 e 2016 em algumas regiões do país (Norte, Nordeste e Centro Oeste com exceção do Distrito Federal), indicando que houve diminuição, por parte das pessoas, de comportamentos de prevenção contra DSTs, nessas regiões.

Na saúde, um dos objetos dos programas sociais é a promoção do sexo seguro. Vários estudos correlatos têm sido realizados sobre, por exemplo, a promoção do uso de preservativo entre jovens (GELUDA et al., 2006), a redução da gravidez indesejada (GALLO, 2011), a análise de fatores associados ao uso de preservativo (MARTINS et al., 2006), além do já citado estudo de Hildebrand e Veloso (2012) sobre o uso de métodos contraceptivos na adolescência. Os resultados desses trabalhos mostram que, apesar do conhecimento dos riscos de não se usar preservativo, jovens de ambos os sexos relatam motivos para não o utilizar: redução de prazer nas relações, parceiro(a) sexual único(a), confiança no(a) parceiro(a) e imprevisibilidade das oportunidades de sexo. Além disso há certamente também a influência de fatores culturais. Por exemplo, um estudo com jovens africanos indica que a opção de não usar preservativo durante 
relações sexuais decorre muitas vezes de crenças que desaprovam o uso (GILES; LIDDELL; BYDAWELL, 2005).

Nos casos em que usar preservativo ou outro comportamento recomendado não é seguido, ou a atitude em relação ao comportamento é negativa, a estratégia de convencimento mais apropriada é a confrontação comportamental e psicológica, com aconselhamento clínico e exposição a comunicações persuasivas para a mudança (SHETH; FRAZIER, 1982).

Comunicações podem persuadir o público-alvo, apresentando ameaças que causem medo (AAKER; WILLIAMS, 1998) ou mesmo empregando humor e ironia (HASTINGS; STEAD; WEBB, 2004) e esperança (THAINIYOM; ELDER, 2017). Os alocutários avaliam os estímulos das campanhas e decidem o tipo de comportamento que devem ter, com bases nas informações assimiladas na comunicação (BLACKWELL; MINIARD; ENGEL, 2005).

A capacidade de persuadir usando as emoções pode ser parcialmente explicada pela neurociência afetiva, que reconhece o papel que as emoções podem ter nos raciocínios morais e nas decisões, assim como sua relativa independência em relação a percursos neurais relacionados com a cognição, atenção, percepção e memória (ALMADA, 2014). A hipótese do marcador somático de Damásio (2012), parte do pressuposto que o sistema de raciocínio evoluiu como extensão do sistema emocional automático, com a emoção desempenhando vários papéis no processo de raciocínio: as respostas desencadeadas pelo cérebro nas estruturas periféricas do corpo, em uma situação emocional, são importantes para trazer informações ao cérebro, retornando como um marcador somático, atraindo a atenção para a tomada de decisão. Dessa forma, os marcadores somáticos poderiam aumentar a eficiência e a precisão do processo de tomada de decisão, gerando sentimentos provocados por emoções (que foram aprendidos pela experiência de vida do sujeito). As sensações corporais advindas de uma situação emocional, poderia reencenar uma situação que já ocorreu no passado, influenciando uma possível tomada de decisão. Por exemplo, a emoção pode dar mais relevo à determinada premissa e assim, influenciar a conclusão em favor dessa premissa, como, por exemplo, o risco de contrair uma DST praticando sexo inseguro. A emoção também auxilia no processo de manter na mente os vários fatos que precisam ser levados em consideração para chegarmos a uma decisão. Como a emoção pode ser ativada inconscientemente, ela pode influenciar processos conscientes de escolha.

Comunicações persuasivas podem mobilizar vários tipos de estímulo. Um dos mais usados é a ameaça, ou seja, a evocação de certa quantidade de medo no receptor da mensagem (HASTINGS; STEAD; WEBB, 2004). Dependendo de sua reação ao medo provocado, o alocutário pode mudar sua atitude e, talvez, posteriormente seu comportamento.

A seguir, são abordados elementos do uso do apelo ao medo e da resposta ao medo em programas de marketing social. 


\section{Uso do apelo ao medo em programas de marketing social}

Apesar de não se conhecer profundamente a relação entre sentimento de medo e motivação para mudança de comportamento (D'SOUZA; TAY 2016), o uso de comunicações com ameaças, que visam despertar o sentimento de medo nas pessoas, é predominante em programas de marketing social (HASTINGS; STEAD; WEBB, 2004), mesmo não havendo a certeza de que tal reação será um motivador ou inibidor de respostas no sentido da autoproteção.

O medo é sentido em situações de perigo iminente ou potencial, contribuindo para a autopreservação do organismo, disparando respostas defensivas (CANOVA; FARIA, 2014).

$\mathrm{Na}$ comunicação, uma ameaça é normalmente composta de mensagens ou imagens mostrando uma situação que o público-alvo deseja evitar, ao passo que a resposta ao medo estimulado é a resposta emocional esperada, que deveria impelir uma pessoa à mudança de atitude ou de comportamento visada (LATOUR; ROTFELD, 1997).

Entre os modelos criados para analisar e predizer como as pessoas reagem a estímulos ameaçadores está o Extended Parallel Process Model (EPPM) (WITTE; ALLEN, 2000; QUICK et al., 2017; JUNG; BRANN, 2014) ou Modelo Estendido do Processo Paralelo. De acordo com o EEPM, uma mensagem com a finalidade de persuadir o público a adotar um comportamento saudável deve induzir: 1) um nível moderado de medo, 2) a percepção de um alto nível de autoeficácia e 3) a identificação de uma resposta eficaz ao risco abordado. Ou seja, o público-reagirá positivamente à mudança de seu comportamento quando avaliar a mensagem como relevante e suscetível de resposta adequada. A intenção é que a pessoa visada na comunicação procure reduzir o risco apresentado via medidas conscientes de mudança de comportamento. Mas, o alocutário pode também reduzir seu medo, negando o risco (pensando: "isso não acontecerá comigo"), fugindo defensivamente ("isso é ameaçador demais; não vou pensar mais nisso") ou reagindo com desconfiança ("estão tentando me manipular, por isso vou ignorar isso") (WITTE; ALLEN, 2000).

Outra vertente de estudos sobre resposta ao medo diante de estímulos ameaçadores aborda as diferenças individuais que afetam a resposta ao medo (por exemplo, MOWEN; HARRIS; BONE, 2004; D'SOUZA; TAY, 2016). O pressuposto principal dessa abordagem é que, dependendo do tipo de situação, diferentes variáveis individuais, como por exemplo, traços de personalidade, são incluídas no processo de avaliação individual de um estímulo e na consequente resposta ao estímulo.

Personalidade é o conjunto habitual de comportamentos, cognições e padrões emocionais que um indivíduo manifesta devido a fatores biológicos e influências ambientais (CORR; MATTHEWS, 2009). Há diversas teorias de personalidade. Nas teorias de traços de personalidade, supõe-se que a personalidade pode ser descrita como conjunto peculiar de traços básicos, combinados em diferentes medidas (FRIEDMAN; SCHUSTACK, 2004). Estudos recentes advindos da neurociência da personalidade, subdisciplina do campo de estudo da personalidade com foco no teste e refinamento de teorias neurobiológicas sobre a personalidade, mostraram que traços de personalidade correspondentes a quatro dos cinco grandes fatores da personalidade - extroversão, amabilidade, neuroticismo e consciência - se associam com o volume de diferentes áreas cerebrais, corroborando o pressuposto de uma base biológica dos traços do modelo dos cinco grandes fatores da personalidade (DeYoung et al. , 2010), mostrando que os traços da personalidade podem influenciar a cognição, emoções e o comportamento. 


\section{Traços de personalidade elementares e modelo proposto para o estudo}

Embora haja muitas teorias de traços de personalidade, a mais importante é a teoria dos Cinco Grandes Fatores da Personalidade (conhecido também como Big Five, Five Factor Model ou simplesmente FFM) que motivou parte da mudança do interesse de estudiosos do comportamento humano por traços de personalidade. De fato, Baumgartner (2002) afirma que o campo de estudos da personalidade foi revigorado no começo da década de $1980 \mathrm{com}$ a elaboração da taxonomia dos cinco fatores da personalidade. O Big Five desvendou a estrutura básica da personalidade, emergindo de um sólido corpo de conhecimento que foi acumulado sobre a estabilidade, hereditariedade, universalidade e utilidade dos traços de personalidade (COSTA e McCRAE, 2008). Flores-Mendoza e Colom (2009) afirmam que o modelo dos cinco fatores da personalidade é o modelo predominante nos estudos da personalidade.

Por serem mais abstratos, os traços elementares de personalidade são mais difíceis de identificar diretamente no comportamento das pessoas. Os cinco fatores de personalidade são Abertura à Experiência, Organização, Introversão, Amabilidade e Neuroticismo (COSTA e McCRAE, 2008).

Nesta pesquisa, o traço de personalidade elementar analisado é a Amabilidade, também denominada Cordialidade ou Afabilidade. Esse traço está relacionado com atitudes e comportamentos pró-sociais. Mowen (200, p. 29) define Amabilidade como "a necessidade de expressar solidariedade e simpatia com os outros." Pessoas com alto grau de Amabilidade apresentam comportamento marcado por cortesia, cooperação e piedade, e tendem a interpretar adequadamente emoções próprias e de outros. Por outro lado, pessoas com baixos escores tendem a ser desconfiadas e gostar de conflitos, além de apresentar comportamentos frios, céticos e egoístas (FRIEDMAN; SCHUSTACK, 2004).

Do exposto, infere-se que pessoas com alto escore em Amabilidade tendem a entender o sofrimento e angústia das pessoas apresentadas nas comunicações, identificando-se empaticamente com elas e, de forma emocionalmente controlada, respondem ao estímulo ameaçador com altos escores de resposta ao medo. Ao contrário, pessoas com baixos escores em Amabilidade, por apresentarem ceticismo, desconfiança e frieza, tendem a apresentar também menores níveis de resposta ao medo, justamente por desconfiar e não acreditar nos argumentos da comunicação.

A relação positiva entre Amabilidade e Resposta ao Medo foi verificada no estudo de Mowen, Harris e Bone (2004), que investigou os antecedentes da resposta ao medo em um anúncio alertando sobre os perigos da direção negligente. Assim, espera-se que indivíduos expostos a mensagens que estimulem medo de se contrair doenças sexualmente transmissíveis na prática de sexo sem proteção apresentem maior percepção de risco por não se usar preservativos. A partir dessa expectativa de resultado, foi formulada a hipótese que pessoas mais amedrontadas pela comunicação tendem a perceber maior risco na atividade sexual insegura (sem preservativo). 
Baseando-se na relação empírica detectada por Mowen, Harris e Bone (2004), elaboramos o modelo da Figura 1, para orientar a pesquisa, propondo as seguintes hipóteses:

Figura 1: Modelo proposto

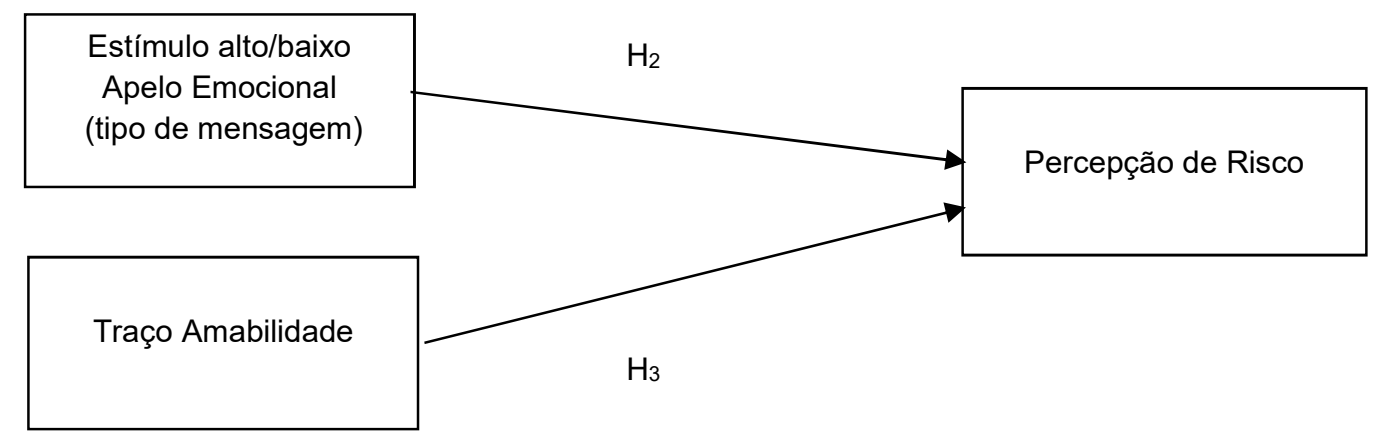

Fonte: Baseado em Mowen, (2000) e Mowen, Harris e Bone (2004).

$\mathrm{H}_{1}$ : (Checagem de manipulação)

"A média de Índice de Medo do grupo experimental é maior do que a média do grupo-controle".

$\mathrm{H}_{2}$ : "Pessoas expostas ao anúncio mais amedrontador tendem a ter maior Percepção de Risco em não usar preservativo'.

$\mathrm{H}_{3}$ : "Pessoas com maior Amabilidade tendem a maior Percepção de Risco em não usar preservativo".

\section{MÉTODO}

Esta pesquisa pode ser classificada como exploratória, com relação aos objetivos, e de natureza quantitativa, quanto ao tipo de análise dos dados. É um estudo quase-experimental, pois aplica parte do aparato experimental, porém sem o controle pleno das variáveis envolvidas. Um estudo quase-experimental, assim como o estudo experimental de laboratório (onde há pleno controle das variáveis), também é composto por unidades de teste, divididas em subamostras homogêneas, variáveis independentes que são manipuladas, variáveis dependentes que são medidas e variáveis estranhas que são controladas (MALHOTRA, 2001).

No quase-experimento, as unidades de teste são alunos de graduação de duas universidades federais do estado de Minas Gerais. A amostra total, escolhida por conveniência, foi composta de 92 alunos, de turmas de diferentes disciplinas, divididos aleatoriamente em grupo-controle $(\mathrm{N}=46)$ e grupo experimental $(\mathrm{N}=46)$. As variáveis independentes foram os escores dos sujeitos no traço Amabilidade (covariável) e o fator manipulado foi tipo de anúncio para alertar sobre o risco de contrair vírus HIV nas relações sexuais e importância preventiva de uso de preservativo. A variável dependente foi a Resposta ao Medo suscitado pelo anúncio. As variáveis estranhas foram parcialmente controladas via procedimento de aplicação do instrumento coleta de 
dados em sala de aula e adoção de intervalo de uma semana entre a exposição aos anúncios e mensuração do traço Amabilidade. No Apêndice, são apresentadas as medidas usadas.

Na manipulação da variável independente, considerou-se como baixo teor de apelo emocional (baixa ameaça) a mensagem impressa exposta ao grupo-controle: "O mundo tem 2,5 milhões de novos casos de Aids por ano. Sexo desprotegido é o meio mais comum de infeç̧ão. Previna-se. Sexo só com camisinha". A outra mensagem, de alto grau de apelo emocional (alta ameaça), exposta ao grupo experimental, continha, além do texto anterior, a imagem de um jovem imunodeprimido expondo os remédios para controlar a doença e ao lado a imagem de outro doente com Aids, mas em estado terminal.

Em sala de aula, após a exposição dos grupos aos anúncios, foi aplicada uma escala para avaliar a resposta ao medo, composta por três medidas, baseada na pesquisa de Mowen, Harris e Bone (2004), ancorada, num extremo, com três diferentes indicações de baixo nível de medo, e noutro, com três níveis elevados de medo correspondentes. A escala utilizada está apresentada no Apêndice, após as Referências. Uma semana depois foi aplicado em sala de aula, o questionário para mensurar o traço de personalidade Amabilidade, nos mesmos 92 alunos, utilizando escala validada e sugerida por Mowen (2000). A mensuração do traço Amabilidade utilizou escalas Likert equilibradas de 7 pontos, ancoradas em "concordo totalmente" e "discordo totalmente". A escala utilizada para mensurar o traço Amabilidade é apresentada no Apêndice deste trabalho.

A Figura 2 mostra o desenho do estudo. A primeira etapa $\left(R, X_{1}, O_{1}, X_{2}, O_{2}\right)$ foi realizada em novembro de 2017. A segunda etapa $\left(\mathrm{O}_{3}\right.$ e $\left.\mathrm{O}_{4}\right)$ foi realizada em dezembro de 2017 , ambas realizadas em sala de aula. Os dados coletados foram verificados e tabulados em planilhas do software de análise estatística SPSS ${ }$. Na análise dos dados foi utilizada a análise de variância de um fator, pois apenas o nível de apelo emocional foi manipulado.

Figura 2: Desenho do quase-experimento

\begin{tabular}{|lllll|}
\hline $\mathrm{GE}$ & $\mathrm{R}$ & $\mathrm{X}_{1}$ & $\mathrm{O}_{1}$ & $\mathrm{O}_{3}$ \\
$\mathrm{GC}$ & $\mathrm{R}$ & $\mathrm{X}_{2}$ & $\mathrm{O}_{2}$ & $\mathrm{O}_{4}$ \\
\hline
\end{tabular}

Nota: $\mathrm{GE}=$ grupo experimental. $\mathrm{GC}=$ grupo-controle. $\mathrm{R}$ = distribuição aleatória. $\mathrm{X}_{1}=$ exposição ao anúncio impresso com texto (baixo teor emocional) sobre a importância do uso de preservativo na prevenção de doenças sexualmente transmissíveis mais imagem de pessoa soropositiva e paciente em estado terminal. $\mathrm{O}_{1}$ : mensuração da variável dependente (Resposta ao Medo) no grupo de tratamento. $\mathrm{X}_{2}$ : exposição ao estímulo na forma de comunicação contendo texto escrito (baixo teor emocional) sobre a importância do uso de preservativo na prevenção de doenças sexualmente transmissíveis. $\mathrm{O}_{2}$ : mensuração da variável dependente (Resposta ao Medo) no grupo de controle. $\mathrm{O}_{3}$ : mensuração do traço Amabilidade no grupo de tratamento. $\mathrm{O}_{4}$ : mensuração do traço Amabilidade no grupo-controle. Fonte: elaborada pelos autores, 2018.

Usa-se ANCOVA para verificar se há diferença de médias entre os grupos (experimental e controle), corrigindo as médias pelas covariáveis na comparação (MALHOTRA, 2001). No trabalho, a variável independente é o grupo (experimental ou controle), a covariável é a Amabilidade e a variável dependente é a Percepção de Risco.

Pressupostos da ANCOVA incluem aleatoriedade da inclusão de indivíduos nos grupos, correspondentes aos fatores manipulados, independência de suas medidas na variável dependente (VD), normalidade da VD para os valores específicos de cada covariável, bem como homogeneidade de variância 
(homocedasticidade) da VD, para cada nível de fator manipulado. Para verificar a normalidade, teste de normalidade deve ser aplicado aos resíduos padronizados. Para testar a homocedasticidade, usa-se o teste de Levene.

\section{ANÁLISES E DISCUSSÕES}

Inicialmente, foram verificadas a unidimensionalidade e confiabilidade das escalas Medo e Amabilidade. Utilizando-se análise fatorial exploratória, extraindo-se fatores com o método de componentes principais, o primeiro fator explicou $89,88 \%$ da variância dos dados na escala de Medo e $62,53 \%$ na escala de Amabilidade, o que nos permite considerá-las unidimensionais (NETEMEYER; BEARDEN; SHARMA, 2003). No teste de confiabilidade, foi calculado o alfa de Cronbach, obtendo-se os valores 0,711 para Amabilidade e 0,944 para Medo, ambos os valores de alfa superiores a 0,700, considerado como patamar mínimo de confiabilidade de medidas unidimensionais (NETEMEYER et al., op. cit.). Verificadas a unidimensionalidade e a confiabilidade das escalas de Medo (soma das respostas das três medidas de medo mostradas) e Amabilidade, foram criados índices somatórios das escalas correspondentes.

Antes de testar o efeito do tratamento experimental na Percepção de Risco de contrair Aids em relações sexuais sem uso de preservativo, fez-se a checagem de manipulação $\left(H_{1}\right)$, comparando-se as médias do Índice de Medo, formada pela soma das notas das três medidas de medo, dos grupos experimental e de controle, aplicando-se teste $t$ para amostras independentes.

Conforme esperado, foi detectada a diferença esperada nos índices de Medo dos grupos controle (M $=14,50, \mathrm{DP}=7,57)$ e experimental $(\mathrm{M}=19,83, \mathrm{DP}=7,03): t(90)=-3,495, \quad p<0,001$, sendo a média na percepção de medo maior no grupo experimental.

Para testar as hipóteses de relação entre Medo e Risco $\left(\mathrm{H}_{2}\right)$ e Amabilidade e Risco $\left(\mathrm{H}_{3}\right)$, foi rodada a ANCOVA.

Admitido o pressuposto de independência das medidas de risco, em função do procedimento de pesquisa, os pressupostos de normalidade e heterogeneidade foram rejeitados, porém de grupos de mesmo tamanho $(N=46)$ e suficientemente grandes $(N>30)$, presumiu-se robustez da ANCOVA, com resultados confiáveis nessas condições (v. por exemplo, resultados de simulações de ROGAN; KESELMAN, 1977, para robustez no caso de heterocedasticidade). Resultados da ANCOVA são mostrados na Tabela 1.

Tabela 1 - Resultados da ANCOVA

\begin{tabular}{lrrrrr}
\hline $\begin{array}{c}\text { Variáveis } \\
\text { Independentes }\end{array}$ & $\begin{array}{c}\text { Soma dos } \\
\text { Quadrados (tipo I) }\end{array}$ & $\begin{array}{c}\text { Graus de } \\
\text { Liberdade }\end{array}$ & $\begin{array}{c}\text { Média dos } \\
\text { Quadrados }\end{array}$ & F & Significância \\
\hline Modelo & $7,426^{(1)}$ & 2 & 3,713 & 4,688 & 0,012 \\
Intercepto & 6818,087 & 1 & 6818,087 & 8608,782 & 0,990 \\
Efeitos principais & 1,410 & 1 & 1,410 & 1,780 & 0,186 \\
Amabilidade & 6,016 & 1 & 6,016 & 7,596 & 0,007 \\
Tipo de Mensagem & & & & &
\end{tabular}

Nota $^{(1)}: R^{2}=0,095$ e $R^{2}$ aj. $=0,075$

Fonte: elaborada pelos autores, 2018. 
O modelo pode ser considerado válido $(F=4,688, p<0,05)$. $O$ valor ajustado de $R^{2}$ indica que a variância explicada da variável dependente (Percepção de Risco) foi de 7,5\%, em função da manipulação do estímulo (Medo) e do traço básico de personalidade (Amabilidade) avaliado dos participantes. Na Tabela 1, o valor significativo do efeito Tipo de Mensagem $(p<0,001)$ atesta a utilidade do estímulo ao medo para conscientizar os jovens sobre a necessidade de uso de preservativo para prevenir a Aids e outras doenças sexualmente transmissíveis, corroborando $\mathrm{H}_{2}$. Porém, não foi detectada a esperada relação entre Amabilidade e Percepção de Risco, levando à rejeição de $\mathrm{H}_{3}$.

\section{CONSIDERAÇÕES FINAIS}

Numa abordagem exploratória, a pesquisa visou verificar se o nível de medo evocado num anúncio impresso e o traço de personalidade Amabilidade podem influenciar a avaliação do risco de não usar preservativo durante relações sexuais. Participantes de um quase-experimento foram submetidos aleatoriamente a uma de duas mensagens: uma de maior apelo emocional para induzir mais medo, com apresentação de texto informativo e imagens (grupo experimental), e outra com menor apelo, composta apenas pelo texto informativo (grupo-controle).

O resultado do estudo empírico sugere que o maior estímulo ao medo pode levar à maior percepção de risco e a um possível comportamento mais precavido, associado ao sexo seguro. Assim, dentro de limites éticos, estimular o medo no público-alvo de contrair uma doença grave, alertando-o para os riscos do comportamento imprudente pode promover o uso de preservativo.

Não se verificou a aventada relação entre Amabilidade e Percepção de Risco, o que nos leva a supor que o poder do estudo realizado tenha sido pequeno para detectar essa relação. Amostras maiores ou estímulos de medo mais forte talvez possam produzir os resultados esperados. Outra possibilidade é que a população universitária já tenha assimilado uma percepção de risco razoável sobre o sexo inseguro, em função do nível educacional dos participantes e da ampla disseminação de informações públicas sobre os riscos de se contrair Aids em relações sexuais sem proteção.

Qualquer que seja a situação real, as autoridades de saúde devem continuar seus programas de promoção do sexo seguro para evitar doenças sexualmente transmissíveis e gravidez não desejada, haja vista o aumento das taxas de incidência de DSTs no Brasil e de casos de gravidez em adolescentes.

Esse estudo apresenta com uma de suas limitações o fato de ser um quase-experimento, em que o controle de variáveis estranhas se limitou ao uso do ambiente de sala de aula, onde há poucas, mas presentes chances de outros fatores interferirem no sentimento de medo evocado pelo estímulo apresentado. Ou seja, não se pode afirmar que houve controle total sobre as variáveis manipuladas. Outra limitação é representada pelo tipo de amostragem adotado. A amostragem por conveniência torna o estudo não probabilístico, por isso não é possível inferir os resultados para a população dos estudantes das Instituições de Ensino Superior analisadas.

O fato de ser um estudo transversal é outra limitação. Os resultados levantados são válidos apenas para o momento em que os dados foram coletados. Para a continuação do estudo do tema, sugere-se a realização de um follow-up em estudo longitudinal, avaliando-se a relação entre os traços de personalidade e a resposta ao estímulo ao medo para a adoção de comportamentos mais prudentes. 


\section{Nota}

1 Modelo desenvolvido nas décadas de 1970 e 1980 por Eysenck, Guilford, McCrae, Costa e Digman, entre outros, a partir dos trabalhos de Klages, Baumgarten e Cattell, publicados na primeira metade do século XX (DIGMAN, 1990).

\section{REFERÊNCIAS}

AAKER, J. L.; WILLIAMS, P. Empathy versus pride: The influence of emotional appeals across cultures. Journal of Consumer Research, 25 (3), 241-261, 1998.

ALMADA, L. A neurociência afetiva como modelo explicativo das emoções básicas. Psicologia Argumento, 32 (79), 2014. ANDREASEN, A. R. Social Marketing: Its definition and Domain. Journal of Public Policy \& Marketing, 13(1), 108-114, 1994.

ANDREASEN, A. R. marketing social Marketing in the Social Change Marketplace. Journal of Public Policy \& Marketing, 21(1), 3-13, 2002.

BAUMGARTNER, H. Toward a Personology of the Consumer. Journal of Consumer Research, 29(2), 286-292, 2002.

BASTOS, A.; COSTA, F.; VASCONCELOS, M.. Consumo de Bebidas Alcoólicas por Jovens: Implicações para o Marketing Social. REMark, 16 (4), 2017.

BLACKWELL, R. D.; MINIARD, P.; ENGEL, J. F. Comportamento do Consumidor. 9. ed. São Paulo: Thomson Learning, 2005.

CANOVA, F.; FARIA, R.S. Proteína Zenk e o condicionamento clássico do medo. Neurociências, 10 (1), 37-47, 2014.

CORR, Philip J.; MATTHEWS, Gerald. The Cambridge Handbook of Personality Psychology (1. publ. ed.). Cambridge, U.K.: Cambridge University Press, 2009.

COSTA, P. T.; McCRAE, R. R. The revised neo personality inventory (neo-pi-r). The SAGE Handbook of Personality Theory and Assessment, 2(2), 179-198, 2008.

CUNHA, B. G. F.; DIAS, M. R. Comunicações persuasivas e doação regular de sangue: um estudo experimental. Cadernos de Saúde Pública, 24, 1407-1418, 2008.

DAMÁSIO, A. O erro de Descartes: emoção, razão e o cérebro humano. Editora Companhia das Letras, 2012.

DEYOUNG, C. G., HIRSH, J. B., SHANE, M. S., PAPADEMETRIS, X., RAJEEVAN, N., \& GRAY, J. R. Testing predictions from personality neuroscience: Brain structure and the big five. Psychological Science, 21(6), 820-828, 2010.

DIGMAN, J. M. Personality structure: Emergence of the five-factor model. Annual Review of Psychology, 41(1), 417-440, 1990.

DONOVAN, R.; HENLEY, N. Principles and practice of social marketing. Cambridge: Cambridge University Press, 2010.

D'SOUZA, C.; TAY, R. Advertising implications and design of messages, Marketing Intelligence \& Planning, 34 (4), $504-$ 522. 2016.

FLORES-MENDOZA, C.; COLOM, R. Introdução à psicologia das diferenças individuais. Artmed Editora, 2009.

FRIEDMAN, H.; SCHUSTACK, M. Teorias da personalidade: da teoria clássica à pesquisa moderna. São Paulo: Prentice Hall, 2004.

GALLO, J. H. S. Gravidez na adolescência: a idade materna, consequências e repercussões. Revista Bioética, 19(1), 179-195, 2011.

GELUDA, K. et al. Quando um não quer, dois não brigam: um estudo sobre o não uso constante de preservativo masculino por adolescentes do Município do Rio de Janeiro, Brasil . Cadernos de Saúde Pública, 22 (8), 1671-1680, 2006.

GILES, M.; LIDDELL, C.; BYDAWELL, M. Condom use in African adolescents: The role of individual and group factors. Aids Care, 17 (6), 729-739, 2005.

GORDON, R.; MCDERMOTTA, L.; STEADA, M.; ANGUSA, K. The effectiveness of social marketing interventions for health improvement: What's the evidence?. Public Health, 120, 1133-1139, 2006.

HAIR JUNIOR, J. F. et al. Análise multivariada de dados. 5. ed. Porto Alegre: Bookman, 2005.

HASTINGS, G.; SAREN, M. The critical contribution of social marketing: Theory and application. Marketing Theory, 3 (3), 305-322, 2003.

HASTINGS, G.; STEAD, M.; WEBB, J. Fear Appeals in Social Marketing: Strategic and Ethical Reasons for Concern. Psychology \& Marketing, 21 (11), 961-986, 2004.

HILDEBRAND, D. F. N.; VELOSO, A. R. Fatores que influenciam o comportamento de consumo de preservativos por adolescentes. Brazilian Business Review, 9, 125-161, 2012. 
JUNG, T.; BRANN, M. Analyzing the extended parallel process model and health belief model constructs in texting while driving: news coverage in leading US news media outlets. International Journal of Health Promotion and Education, 52(4), p. 210-221, 2014.

KOTLER, P.; LEE, N. Marketing no setor público: um guia para um desempenho mais eficaz. Porto Alegre: Bookman, 2008.

KOTLER, P.; ZALTMAN, G. Social Marketing: An Approach to Planned Social Change. Journal of Marketing, 35, 3$12,1971$.

LATOUR, M. S.; ROTFELD, H. J. There are Threats and (Maybe) Fear-Caused Arousal: Theory and Confusions of Appeals to Fear and Fear Arousal Itself. Journal of Advertining, 26, (3), 45-59, 1997.

LAI, PO-HSIN et al. Integrating social marketing into sustainable resource management at Padre Island National Seashore: an attitude-based segmentation approach. Environmental management, 43 (6), 985-998, 2009.

LEFEBVRE, R. C. Social marketing and social change : strategies and tools for health, well-being, and the environment. 1. ed. San Francisco: Jossey-Bass, 2013.

MALHOTRA, N. Pesquisa de Marketing: uma orientação aplicada. 3. ed. Porto Alegre: Bookman, 2001.

MARTINS, L. B. M. et al. Fatores associados ao uso de preservativo masculino e ao conhecimento sobre DST/ Aids em adolescentes de escolas públicas e privadas do Município de São Paulo, Brasil. Cadernos de Saúde Pública, 22 (2), 315323, 2006

MEIRA, P. R. S.; SANTOS, C. P. Programas de marketing social: proposição e exame de uma estrutura conceitual de avaliação de resultados. Revista de administração pública, 46, (2), 493-522, 2012.

MINISTÉRIO DA SAÚDE. Boletim epidemiológico HIV - Aids. . [S.I.]: Ministério da Saúde, Secretaria de Vigilância em Saúde - Departamento de DST, Aids e Hepatites Virais, 2017. Disponível em <http://www.aids.gov.br/ptbr/pub/2017/boletim-epidemiologico-hivaids-2017> .

MOTA, J. R. et al. Impacto do apelo ao medo nas embalagens do cigarro: a percepção de fumantes em relação às mensagens de advertência antitabagismo. Revista Eletrônica de Ciência Administrativa, 12 (2), 112-123, 2013.

MOWEN, J. C. The 3M model of motivation and personality: theory and empirical applications to consumer behavior. New York: Springer Science+Business Media, 2000.

MOWEN, J. C.; HARRIS, E. G.; BONE, S. A. Personality traits and fear response to print advertisements: theory and an empirical study. Psychology \& Marketing, 21 (11), 927-943, 2004.

NETEMEYER, R. G.; BEARDEN, W. O.; SHARMA, S. Scaling Procedures: Issues and Applications. Sage Publications, 2003.

PEATTIE, Ken; PEATTIE, Sue. Social marketing: A pathway to consumption reduction?. Journal of Business Research, 62 (2), 260-268, 2009.

QUICK, B. L.; LAVOIE, N. R.; REYNOLDS-TYLUS, T.; MARTINEZ-GONZALEZ, A.; SKURKA, C. Examining Mechanisms Underlying Fear-Control in the Extended Parallel Process Model. Health Communication, 1-13, 2017.

ROCHA, J. C. et al. Panorama Nacional de Publicações em marketing social na Administração.Revista de Administração IMED, 7 (2), 227-253,2017. doi:https://doi.org/10.18256/2237-7956.2017.v7i2.2076.

ROGAN, J. C.; KESELMAN, H. J. Is the ANOVA F-test robust to variance heterogeneity when sample sizes are equal?: An investigation via a coefficient of variation. American Educational Research Journal, 14(4), 493-498, 1977.

RUCKER, D. D.; PETTY, R. E. Increasing the Effectiveness of Communications to Consumers: Recommendations Based on Elaboration Likelihood and Attitude Certainty Perspectives. Journal of Public Policy \& Marketing, 25(1), 39-52, 2006.

SAGAZ, S. M.; LUCIETTO, D. A. marketing social Aplicado à Saúde Coletiva: Definições, Usos, Aplicações e Indicadores da Produção Científica Brasileira. Revista Pensamento Contemporâneo em Administração, 10(3), 16-30, 2016.

SCHNEIDER, G; LUCE, F. B. Marketing social: abordagem histórica e desafios contemporâneos. Revista Brasileira de Marketing, 13(3), 125-137. 2014

SHETH, J. N.; FRAZIER, G. L. A Model of Strategy Mix Choice for Planned Social Change. Journal of Marketing, 46 (1), 15-26, 1982.

THAINIYOM, P.; ELDER, K. Emotional Appeals in HIV Prevention Campaigns: Unintended Stigma Effects. American Journal of Health Behaviour, 41(4), 390-400, 2017.

WETTSTEIN, D.; SUZANNE SUGGS, L.; LELLIG, C. Social marketing and alcohol misuse prevention in German-speaking countries. Journal of Social Marketing, 2 (3), 187-206, 2012.

WITTE, K.; ALLEN, M. A Meta-Analysis of Fear Appeals: Implications for Effective Public Health Campaigns. Health Education \& Behavior, 27(5), 591-615, 2000. 